\title{
Implicaciones teóricas, politicas y metodológicas de la investigación activista feminista
}

\author{
Theoretical, political and methodological implications of \\ feminist activist research.
}

\author{
Ana María Castro Sánchez \\ Universidad del Tolima en Colombia \\ amcastros@ut.edu.co (COLOMBIA)
}

Recibido: 26.062019

Aceptado: 10.03 .2021

\section{RESUMEN}

La reflexión sobre la relación entre la investigación y el activismo es significativa para repensar los procesos de construcción de conocimientos y sus implicaciones en diferentes niveles. Nociones tradicionales y hegemónicas del conocimiento lo ubican en un lugar separado de la realidad que supuestamente pretende descubrir y explicar, lo cual lo aleja de objetivos políticos y de la necesidad de otro tipo de prácticas o acciones que lo retroalimenten. Este texto aborda las implicaciones teóricas, políticas y metodológicas que trae consigo la realización de investigaciones activistas, particularmente desde una perspectiva feminista, teniendo en cuenta que estas implicaciones no se encuentran separadas, por el contrario las decisiones y los modos de hacer en cada uno de estos ámbitos repercute en los otros, en una relación dialéctica.

\section{PALABRAS CLAVE}

Investigación activista, investigación activista feminista, metodología, política, activismo.

\section{ABSTRACT}

The reflection on the relationship between research and activism is significant in order to rethink the processes of knowledge construction and its implications at different levels. Traditional and hegemonic notions of knowledge place it in a place separate from the reality that supposedly seeks to discover and 
explain, which distances it from political objectives and the need for another type of practice or action that feed it back. This text deals with the theoretical, political and methodological implications of activist research, particularly from a feminist perspective, taking into account that these implications are not separated, on the contrary, the decisions and ways of doing in each one of them have an impact on the others in a dialectical relationship.

\section{KEY WORDS}

Activist research, feminist activist research, methodology, politics, activism.

No se trata simplemente de hacer investigación para encontrar respuestas o trazar caminos a seguir. Más bien, la habilidad de investigar y el hecho investigativo constituyen el reconocimiento de que no existen respuestas claras, unilaterales o universales. Ésa es la base de la acción política y ética actual.

María Isabel Casas-Cortés, Michal Osterweil y Dana Powell

\section{INTRODUCCIÓN}

La reflexión sobre la relación entre la investigación y el activismo es significativa para repensar los procesos de construcción de conocimientos y sus implicaciones en diferentes niveles. Nociones tradicionales y hegemónicas del conocimiento lo ubican en un lugar separado de la realidad que supuestamente pretende descubrir y explicar, lo cual lo aleja de objetivos políticos y de la necesidad de otro tipo de prácticas o acciones que lo retroalimenten. Sin embargo, hoy en día es reconocida la carga de poder que la producción de conocimiento trae consigo, de allí los cuestionamientos de las prácticas académicas y la necesidad que éstas se comprometan y articulen con prácticas de activismo político haciéndolas más flexibles, abiertas (Leyva 2010), y por lo tanto más pertinentes.

Estas propuestas se concretan en las denominadas investigaciones activistas, donde han tenido particular relevancia las que se postulan desde perspectivas políticas feministas. Así se da lugar a las investigaciones activistas feministas, que buscan poner en práctica tanto las propuestas de las epistemologías feministas como metodologías, métodos y técnicas de investigación que sean consecuentes. Para comprender estos aportes analizaré las implicaciones que trae consigo la realización de investigaciones activistas desde una perspectiva feminista en los aspectos teóricos, políticos y metodológicos, sobre este punto en particular me referiré a las producciones narrativas ${ }^{1}$ como propuesta metodológica desde

\footnotetext{
${ }^{1}$ En este acápite retomaré algunos aspectos que son producto de mi experiencia en la realiza-
} 
la epistemología de los conocimientos situados. Si bien estos tres aspectos son interdependientes en este texto los presentaré de forma separada, con el interés de facilitar la comprensión y vislumbrar sus vínculos.

\section{IMPLICACIONES TEÓRICAS}

Las diversas consideraciones sobre cómo se producen los conocimientos, las influencias que se ejercen sobre los mismos y sus repercusiones han sido abordadas desde diversas perspectivas. Una de ellas ha sido la epistemología crítica que plantea alternativas a las nociones imperantes en relación a la producción de conocimientos. Norma Blazquez (2012), siguiendo el análisis de Helen Longino, señala que tanto los prejuicios personales, como los intereses políticos y sociales impactan la producción de conocimiento, incluso el denominado científico, aunque éste se imponga como neutral, objetivo, universal y racional. Las prioridades que se establecen en una investigación relacionadas con preguntas, temas, marcos teóricos, explicaciones, métodos, datos -válidos o invalidados-, interpretaciones, comparaciones, conclusiones, recomendaciones, entre otros aspectos, son afectados por dichos prejuicios, intereses, o juicios que dependen de suposiciones que pueden estar influidas por valores y creencias individuales, culturales y científicas, al ser no solo las estrategias de investigación, sino el lenguaje de la ciencia e incluso la tecnología construcciones humanas.

Esta epistemología que es crítica con los pilares de la ciencia moderna, con la propia noción de conocimiento, ha llevado a autorxs a plantear la existencia de una violencia epistémica que también impacta en la definición de quién puede hacer ciencia y cuáles experiencias pueden ser o no consideradas como conocimiento. Se constituye así una "instancia de patrullaje" que permanece oculta dentro de la academia dominante, lo que lleva a que el carácter político del conocimiento sea invisibilizado bajo el resguardo de la objetividad científica (Suárez-Krabbe 2011: 196).

En el marco de esta mirada, que incluye la definición de lxs sujetos de conocimiento y las experiencias que lo constituyen, las investigaciones activistas han ratificado que son posibles epistemologías que procuren otros sistemas de conocimiento alternativos a la ciencia hegemónica occidental. Abarcan así el debate sobre el tipo de academia que se considera legítima, a qué intereses responde y cuál es su lugar en el sistema mundo/moderno colonial (Leyva 2010), de allí la propuesta de estar siempre alerta frente a qué investigación se produce, para qué y para quién se produce el conocimiento.

Las investigaciones activistas confirman, en el ejercicio concreto del quehacer investigativo, que los aspectos teóricos no están separados de los políticos. Gracias a estas miradas críticas hoy comprendemos que todas las investigaciones, sin importar el área, la disciplina, los enfoques metodológicos y, por supues-

ción de una investigación activista feminista, cuyo resultado fue mi tesis doctoral "Arte con política en el activismo feminista. Narrativas de la acción política revuelta" (2018). 
to, las perspectivas teóricas tienen implicaciones y efectos políticos, la diferencia radica en que no todas las investigaciones los hacen explícitos y las que son reflexivas frente a sus implicaciones actúan en consecuencia.

En este sentido, lo que distingue a las investigaciones activistas es el cuestionamiento sobre las implicaciones políticas en relación a cómo y qué tipo de conocimiento producimos, qué objetos se estudian, cómo se hacen las investigaciones, qué significan para los colectivos, con quién y cómo se trabaja. Ello deviene de la necesidad de superar la distancia que se ha intentado sostener entre teoría y práctica, entre trabajo intelectual y activismo político, entre investigación e intervención social; distancia que llevada al extremo por una parte subvalora el trabajo en el "mundo real" y de otra elude la importancia de la teoría.

Shannon Speed, desde su postura como antropóloga ${ }^{2}$, afirma que la investigación activista se refiere a

"a un compromiso franco para involucrarnos con nuestros sujetos de estudio de manera que compartamos metas políticas. Lo que quiero argumentar, y es la razón por la que uso el término, es que ambos, la investigación y el activismo, pueden ser practicados simultáneamente de forma productiva como componentes de un mismo esfuerzo. Este tipo de investigación es necesariamente colaborativa y establece el diálogo entre antropólogos[as] y aquellos[as] con quienes trabajamos a través de un proceso de investigación" (Speed 2011: 276)

Speed diferencia entre el compromiso de una investigación y el compromiso activista, sin desconocer la tensión que se puede presentar entre el análisis crítico y el compromiso ético y político, tensiones que están presentes en todas las investigaciones pero que, en el caso de las activistas, van a ser centrales incluyéndolas como tema de trabajo. Otro aspecto relevante de los vínculos que se establecen entre activismo e investigación, es que están "relacionados a partir del reconocimiento de que lo personal es político, encontrando allí su enclave feminista ya que no se propone que las investigaciones distingan entre espacios públicos y privados, entre sujetos y objetos, razón y emoción y otros dualismos, sino que estos están interrelacionados y se influencian de manera continua" (Fulladosa-Leal 2015: 137).

Por ello, para las investigaciones activistas también han sido fundamentales los planteamientos de las epistemologías feministas, en la medida en que éstas examinan la carga androcéntrica, sexista, racista, clasista y heteronormativa de los fundamentos sobre los que se construye el denominado conocimiento científico, develando sus sesgos, las cargas ideológicas, las relaciones de poder, y lo que subyace en los criterios sobre los cuales se valora lo que puede ser o no considerado conocimiento.

${ }^{2}$ Los aportes que han hecho a las epistemologías críticas lxs antropologxs que hacen investigación activista son relevantes, en la medida en que la antropología ha sido una de las ciencias que más ha objetivado a los sujetos de investigación, con sus metodologías de características coloniales. 
Asimismo, las epistemologías feministas han analizado críticamente el lugar de lxs sujetxs productores de conocimiento, haciendo visibles los diferentes intereses, las relaciones sociales sobre las cuales se produce y está constituido el conocimiento, los elementos subjetivos que también son determinantes, los contextos institucionales de producción de conocimientos, además de las implicaciones de los diferentes privilegios que ostentan quienes hacen parte de dichos contextos. "La reflexión feminista ha aportado una visión del conocimiento como práctica social desde donde poder recrear procesos de investigación hilando reflexión y práctica" (Fulladosa-Leal 2015: 117), ampliando así la perspectiva de la epistemología crítica y las implicaciones del compromiso activista.

La investigación activista que asume estas propuestas se ha denominado como investigación activista feminista ${ }^{3}$, su característica es que pone en práctica las propuestas de las epistemologías feministas, al contrastar las interpretaciones de la realidad con las que se encuentran en las teorías que intentan explicarla, procediendo de lo concreto a lo abstracto, para volver a lo concreto en forma de praxis en la búsqueda de transformaciones; de allí surge la necesidad de la valoración de las experiencias, las acciones y las prácticas. Igualmente, asume las críticas epistemológicas feministas que superan el marco epistemológico hegemónico al producir saberes políticos colectivos, ya que no se trata de buscar nuevas leyes para identificar la ciencia, sino evidenciar la reconstrucción de los procesos de negociación que implican las construcciones y representaciones humanas y sus relaciones de poder (Biglia 2012: 198).

De aquí deviene la crítica a la teoría que se presenta como neutral y desencarnada, posicionada desde un lugar en el que afirma ver todo pero realmente es solo una parte, es el "testigo modesto" del que nos habla Donna Haraway (2004), donde la modestia es la característica, entre otras subjetiva, que permite eliminar la atención sobre el sujeto y las incómodas preguntas por sus intereses y posicionamientos. La crítica a este "testigo" se debe justamente a que el conocimiento pasa por el cuerpo, está encarnado, incorporado, por lo cual es un pensamiento implicado, situado, parcial. Ello responde a la certeza que toda producción de conocimiento afecta los cuerpos, las vidas, la subjetividad de quienes participan en dichos procesos de construcción. Para Marta Malo (2004: 34-35), otros tipos de producción de conocimiento pueden construir otros cuerpos, así como otras prácticas que se potencian y valoran de maneras distintas, de allí que no sea separable el proceso de construcción de conocimiento del proceso de construcción de subjetividades.

Es por ello que la investigación activista feminista parte de la convicción de que los conocimientos son parciales, inacabados y tienen una clara intencionalidad política. Esta postura contrasta contra la idea hegemónica del conocimiento acabado, completo, neutral, que difiere con la realidad de un mundo caracteriza-

${ }^{3}$ A pesar de ser reconocida la importancia e influencia de las epistemologías y las políticas feministas, no todas las investigaciones activistas se plantean desde una postura feminista que ponga en práctica las propuestas mencionadas, de allí la importancia de mencionarlas como investigaciones activistas feministas, para no caer en el uso del feminismo solo como un adjetivo. 
do ciertamente, como afirma Santos (2006), por una diversidad epistemológica, ontológica y cultural, además:

"a diversidade epistemológica não é um mero reflexo ou epifenómeno da diversidade ou heterogeneidade ontológica. Ela assenta na impossibilidade de identificar uma forma essencial ou definitiva de descrever, ordenar e classificar processos, entidades e relações no mundo. O própio acto de connhecer [...] é uma intervenção sobre o mundo, que nos coloca neste e aumenta a sua heterogeneidade. Diferentes modos de conhecer, sendo necessariamente parciais e situados, terão consequências diferentes e efeitos distintos sobre o mundo" (Santos, 2006:37).

La investigación activista feminista reconoce que los efectos y las consecuencias que tienen los diferentes modos de conocer que menciona Santos, son diferenciales para las mujeres y para los hombres, o para quienes no se identifican con ninguna de estas dos categorías, indicando la complejidad del binarismo de género como dispositivo de captura. Además, no solo se debe tener en cuenta la condición y posición de género, sino las interrelaciones con la clase, las etnias, las orientaciones sexuales, la edad y demás marcas identitarias y sistemas de opresión imbricados que también van a repercutir en las implicaciones del conocimiento, sus usos, potencialidades y límites.

Es así que la propuesta del conocimiento situado se constituye para la investigación activista feminista en un reto, pues es necesario pasar de la retórica a la puesta en práctica de investigaciones realmente situadas. No se trata solo de nombrar nuestras posiciones y lo que nos condiciona y determina, sino analizar cómo ello influye concretamente en la construcción de conocimientos, en nuestras formas de hacer. Apuesta cuya complejidad e importancia muchas veces se ha minimizado, lo que se explica según Biglia y Jimenéz por:

"En primer lugar, la importancia que se atribuye al lenguaje posmoderno en la academia que genera la impresión de que, en lugar de poner en práctica las enseñanzas de los conocimientos situados, es suficiente con narrarlas, eso sí, con terminologías complejas y altisonantes. En segundo lugar, el hecho de que las teorías críticas están relativamente de moda y actualmente es políticamente correcto hacer referencia a algunos aspectos de las teorías feministas, elementos que han conllevado a la producción de muchos trabajos despolitizados dentro de estas áreas. Por último, la falta de reflexiones sistematizadas y corporeizadas sobre cómo asumir estos posicionamientos" (Biglia y Jimenéz 2012: 111).

Se trata por tanto de aprender a construir conocimientos situados, lo que implica no solo pensar la posición de quien investiga para rehacer su rol, sino identificar las implicaciones del posicionamiento de las diferentes personas con las cuales se construye conocimiento y la manera como estas posiciones entran en relación. Esto no debe ser solo un momento específico, por ejemplo, cuando se inicia la investigación, porque allí nos quedaríamos solamente en el nombrar, sino que debe ser una reflexión constante en todos los momentos que constituyen el proceso de esta construcción, para poder identificar las resonancias e impli- 
caciones que nuestras posturas situadas van teniendo y las respuestas que a ello se deben dar.

En relación a la importancia de situarnos, Shannon Speed (2011: 291-292) reflexiona en torno a la percepción de la naturaleza socialmente situada de nuestra producción de conocimiento, a partir de la cual es necesario "incorporar una consideración reflexiva sobre la forma en que nuestro posicionamiento afecta el conocimiento que producimos. Esto incluye consideraciones sobre nuestro poder y autoridad en la relación que establecemos con nuestro sujeto de estudio". Para la autora, lo que deriva de este conocimiento situado es hacer explícitas las alianzas de la investigación activista, la claridad en los compromisos políticos y el diálogo abierto con quienes establecemos relaciones de construcción de conocimiento.

Para que una investigación sea activista necesita de procesos colectivos de construcción de conocimiento desde diferentes lugares, miradas, sujetxs, lenguajes, relaciones. Mi experiencia en este sentido es que para lograrlo es necesario aprender a tramitar el lugar de investigadorx, que permita construir los puentes necesarios con lxs sujetxs/actores involucradxs; así como hacer más explícita la posición situada, que no solo está determinada por las marcas identitarias sino también por lo que elegimos hacer y desde dónde mirar.

En la escritura final del texto, producto del ejercicio de investigación activista feminista, viví un conflicto relacionado con las reflexiones planteadas por las sujetas de la investigación con las que no estaba de acuerdo, momentos donde consideraba que su saber necesitaba una lectura crítica desde mi posición; así comprendí el postulado de que no es posible, ni es necesario, ser neutral como lo postulan las epistemologías feministas. Este conflicto estaba relacionado con la importancia que le dí en el proceso al respeto y cuidado de las sujetas de investigación, como una postura ética y de valoración de sus conocimientos. Esto refleja también que las dinámicas de poder no pueden ser negadas en las investigaciones, lo importante es repensar el ejercicio de poder que se ejerce y cómo gestionarlo.

Otra de las características de las investigaciones activistas feministas, que se suma a la puesta en práctica del pensamiento y los conocimientos situados, es que postulan la necesidad de superar la fragmentación ficticia que se ha construido entre conocimiento teórico y práctico, entre un conocimiento ajeno a la vida de las personas y uno que tenga sentido para la vida; de allí la importancia de repensar desde dónde es posible construir conocimientos, cómo se pueden producir, a qué otras lógicas pueden responder. En este sentido Xochitl Leyva (2010) afirma que actualmente es posible reconocer que se han ido construyendo otros tipos de conocimiento dentro de las dinámicas propias de los movimientos políticos, de los que son expresión y que como tal están en permanente construcción.

Para Casas-Cortés, Osterweil y Powell, dichos conocimientos se desarrollan a través de diferentes prácticas de conocimiento e incluyen:

"por un lado, análisis, conceptos, teorías, imaginarios -incluyendo las categorías mismas de identificación colectiva y análisis político a partir de 
las cuales actúan- y, por otro lado, artefactos metodológicos y herramientas de investigación. Además, también comprenden prácticas asociadas, de una forma menos obvia, con el conocimiento, incluyendo la generación de subjetividades/identidades, discursos, sentido común y proyectos de autonomía y de vida" (Casas-Cortés, Osterweil y Powell 2010: 8).

Este planteamiento se articula con el de Leyva (2010), en el sentido de que estos otros tipos de conocimiento no se pueden encasillar solo como activistas o académicos, ya que se están produciendo colectivamente, con la participación activa de académicxs, que repiensan su lugar y reconstruyen las relaciones tradicionales impuestas sobre el sujeto y el denominado objeto de la investigación, lo que caracteriza los conocimientos producto de estas relaciones como activistas. Asimismo, dichas relaciones se transforman a partir del mutuo reconocimiento de los diferentes aportes que cada persona puede compartir en una construcción colectiva de conocimientos, que se nutre tanto de perspectivas teóricas como de experiencias, de las reflexiones que éstas suscitan, del encuentro entre diferentes miradas y formas de ser y estar en el mundo, de la constante reflexión sobre los conocimientos que se construyen.

Xochitl Leyva (2010) nos convoca a comprender que otra de las particularidades que se pueden reconocer en estos conocimientos, que son resultado precisamente de investigaciones activistas, es que responden a más de una lógica, se expresan en más de una gramática, lenguaje y lengua. De esta manera se supera la imposición del conocimiento hegemónico en relación a los usos del lenguaje, las maneras de escritura y expresión que constituyen un canon, que no solo reduce sino que desconoce la potencia comunicativa, explicativa y propositiva de la diversidad de lógicas, lenguajes, lenguas y gramáticas que existen.

Asimismo, la investigación activista implica una explícita y abierta reflexión colectiva sobre el propio proceso de producción de conocimiento, reflexión que en ocasiones es marginalizada y subvalorada por manifestar abiertamente las contradicciones, vacíos y sesgos del conocimiento académico, por lo cual, como sostiene Leyva (2010), incluso se le percibe como un peligro para las instituciones académicas.

Este tipo de apuestas pueden ser posibles justamente en investigaciones activistas feministas, donde no se trata de construir conocimientos de o sobre un objeto de estudio, sino junto, con y para lxs sujetos con los que se establecen relaciones de conocimiento. Se trata por tanto de "reconocer a las personas con quienes investigamos como sujetas de pensamiento y creadoras de teoría feminista [lo que] supone, para quien investiga, cuestionarse el lugar que ocupa en el mapa de relaciones de poder que han generado las ciencias sociales y, por tanto, dejar de investigar "sobre" para investigar "con" (Medina 2019: 119).

De allí que las experiencias más relevantes sean las que se han desarrollado articulando prácticas académicas con activismos políticos, particularmente movimientos sociales, con las cuales se busca producir saberes políticos colectivos, sumadas a otras búsquedas entre:

"el pensamiento, la acción y la enunciación: iniciativas que se preguntan 
cómo romper con los filtros ideológicos y los marcos heredados, cómo producir conocimiento que beba directamente del análisis concreto del territorio de vida y cooperación y de las experiencias de malestar y rebeldía, cómo poner a funcionar este conocimiento para la transformación social, cómo hacer operativos los saberes que ya circulan por las propias redes, cómo potenciarlos y articularlos con la práctica [...] en definitiva, cómo sustraer nuestras capacidades mentales, nuestro intelecto, de las dinámicas de trabajo, de producción de beneficio y/o gobernabilidad, y aliarlas con la acción colectiva (subversiva, transformadora), encaminándolas al encuentro con el acontecimiento creativo" (Malo 2004: 15).

Las transformaciones que a nivel teórico ha suscitado la investigación activista, se reconocen en experiencias de producción de conocimientos sobre los mecanismos de dominación, en las cuales se potencian saberes considerados menores -que se producen colectivamente- en contraste con saberes catalogados como expertos -producidos individual y privadamente-. En una perspectiva similar de análisis, en el desarrollo de sus trabajos de investigación activista con diversos movimientos sociales, María Isabel Casas-Cortés, Michal Osterweil y Dana Powell (2010), proponen reconocer como prácticas de conocimiento - "knowledge-practices"- los saberes producidos en los movimientos sociales como parte fundamental de sus dinámicas cotidianas, reconocidas como espacios de creación, reformulación y difusión de conocimientos.

Esta categoría, intenta evitar las connotaciones abstractas normalmente asociadas con el conocimiento y destacar su carácter concreto, corporizado, vivido y situado. Estos conocimientos, creados, modificados y puestos en escena de manera creativa pueden ser reconocidos tanto en las historias, ideas, narrativas e ideologías de los movimientos sociales. Asimismo, pueden tomar la forma de teorías, conocimientos expertos, así como análisis políticos y comprensiones críticas de contextos particulares. Ello incluye tanto aspectos tradicionales del conocimiento como competencias científicas o intervenciones en el nivel micropolítico y cultural relacionadas principalmente con el conocimiento concebido como "saber hacer".

Siguiendo a Casas-Cortés, Osterweil y Powell (2010: 28), las prácticas de conocimiento tienen una potencialidad importante a nivel teórico en la medida en que actúan a partir de concepciones críticas del mundo que posibilitan un acceso al análisis y teorías sociopolíticas que resultan únicas por ser coyunturales, estar claramente localizadas y situadas en procesos de lucha. Por tanto, es un conocimiento que interactúa con expresiones del poder, a partir de las cuales se construyen diversas visiones sobre alternativas y posibilidades de cambios sociales que con dificultad podrían propiciarse desde otros lugares de producción de conocimientos ajenos a las militancias o activismos. Otro aspecto importante que resaltan las autoras es que las prácticas de conocimiento, al ser forjadas en campos de poder, se confrontan con los regímenes epistémicos y ontológicos que los movimientos sociales buscan transformar "sea a través de la oposición directa y explícita a discursos "expertos" o bien por medio de la proliferación de una variedad de modos alternos de saber y ser". 
Para la investigación activista feminista esta propuesta de la producción de conocimientos, que se encuentra en la cotidianidad de los movimientos sociales, puede ser una herramienta útil, ya que, además de hacer visible la diversidad de formas, usos y efectos de estas prácticas, pone en cuestión los significados y el uso mismo de lo que se considera conocimiento.

Construir conocimientos teniendo claro los objetivos políticos y, por ello, activistas, implica remover la idea preponderante de la investigación que se centra y cierra en sí misma, lo que lleva a preguntarnos sobre "cómo orientarnos en la investigación sin quedar atrapados en la seducción paralizante de las grandes teorías o, en su defecto, en la obsesión descriptiva propia de la mirada etnográfica o del puro empirismo sociológico" (Svampa 2008: 166). Se trata, por tanto, de reconsiderar el lugar de la teoría para romper el círculo reafirmatorio del que habla Feyerabend (1977), cuando estamos constantemente en la búsqueda de datos que demuestren las teorías o que manipulamos de tal manera que los hacemos coincidir. De allí su propuesta de proceder contrainductivamente para confrontar las teorías y hacer visibles sus componentes ideológicos, que podemos contrastar y examinar para hacer evidentes otros puntos de vista nuevos, no comunes y disponer así de otras interpretaciones de la realidad que reconozcan, hagan visibles y revaliden las diversas experiencias.

\section{IMPLICACIONES METODOLÓGICAS}

Si las implicaciones teóricas de la investigación activista feminista tienen que ver con la diversidad de conocimientos y las múltiples maneras de construirlos, en consonancia con la acción política y mas allá de los esquemas restringidos del ámbito académico y del canon de la ciencia occidental moderno/ colonial, la pregunta que surge es cómo hacerlo. Al respecto, las epistemologías feministas han llamado la atención sobre las dificultades en relación a los referentes metodológicos que puedan ser considerados feministas, ya que

"Los retos abiertos por las epistemologías feministas son más fácilmente abarcables en el plano teórico que en el empírico [...] realizar la investigación coherentemente con las propuestas epistemológicas feministas por falta de formación se hacen aún más extremas por la necesidad de justificación de la misma a la hora de plantear temas y enfoques que rompen la lógica patriarcal de la ciencia” (Biglia y Jimenez 2012: 107-108).

$\mathrm{Al}$ igual que las epistemologías, las metodologías que se posicionan desde un enfoque feminista y activista, no son únicas o estáticas, por el contrario están en constante construcción. De allí que sea posible el uso de diferentes metodologías aplicadas incluso en otro tipo de investigaciones, la diferencia está en los criterios con los cuales se opta por alguna de ellas, por determinados métodos y por el uso crítico de las técnicas de investigación posibles de ser recreadas, deconstruidas o innovadas. Una alerta importante en este sentido es no reducir la complejidad que esto implica a creer que se esta haciendo investigación activista 
feminista porque se elige un campo de estudio o temática que consideramos relacionada con un género o sus conflictos, ya que por el contrario se estarían reiterando los estereotipos y el orden de género, así como reproduciendo métodos de manera acrítica (Biglia 2012).

Asimismo, Bárbara Biglia (2012) insiste en la importancia de no caer en la confusión que aún hoy en día encontramos en las investigaciones, entre utilizar metodologías feministas, asumir una perspectiva de género, investigar "sobre" temas relacionados con lo construido social, cultural e históricamente como femenino o trabajos que se realizan exclusivamente con mujeres. Al respecto es necesario insistir en que cualquier objeto o sujeto de estudio es susceptible de una investigación no sexista, como ya lo han demostrado las feministas que trabajan en todas las áreas del conocimiento, puesto que "todo tema es tema del feminismo" (Harding 1998: 19).

Por tanto, no hay una preocupación por definir un método feminista a manera de receta, pues sería contradictorio con las epistemologías feministas, ya que la apuesta está en los encuentros y posibles combinaciones críticas entre diferentes metodologías, métodos y técnicas de investigación con otras perspectivas de análisis y posturas políticas. De esta manera, más que delimitar se pueden caracterizar las metodologías implementadas en las investigaciones activistas feministas como campos de formación flexibles, dinámicos y contingentes. Teniendo en cuenta estas concepciones, son pertinentes las aproximaciones mutimetodológicas para abordar los problemas de investigación desde perspectivas feministas activistas, las cuales suponen, siguiendo a Castañeda (2008), que no hay una normatividad metodológica aplicada acríticamente, y que las elecciones en el ámbito metodológico conciernen a los contextos, las experiencias y las orientaciones epistemológicas.

En este sentido, es central en la apuesta metodológica de las investigaciones activistas feministas la coherencia con los procesos y los objetivos que se proponen y no solo el uso de cualquier método y sus técnicas previamente formalizadas, ya que:

"El método, abstraído del contexto y de las preocupaciones de las que nace, se convierte en un corsé que impide la verdadera conexión entre experiencia y pensamiento, entre análisis y práctica de transformación, una especie de rejilla ideológica que atora los desplazamientos ante los nuevos problemas e inquietudes que el proceso va planteando a medida que avanza. Por encima de cualquier método, están las operaciones reales que el proceso de investigación militante es capaz de poner en marcha. La investigación militante es, en este sentido, siempre, un viaje abierto, que sabemos de dónde y cómo parte pero no a dónde nos llevará" (Malo 2004: 35).

Este interés por pensar los métodos de investigación y no solo aplicarlos obcecadamente tiene resonancia con las propuestas de Jhon Law (2004), cuando afirma que los métodos son limitantes ya que están pensados para un repertorio restringido de respuestas y por ello terminan siendo normativos, en una especie de hegemonía metodológica que no es posible adaptar al estudio de lo irregular, lo efímero, lo indefinido que es la realidad, imponiendo reglas sobre cómo 
debemos ver y lo que debemos hacer, preceptos que si no seguimos tienen la consecuencia de no estar "conociendo bien".

Law afirma además que los métodos no solo describen sino que producen la realidad que pretenden comprender y explicar. Por ello, es posible pensar diferentes métodos que no busquen algo definido, repetible o estable, lo que implica deshacer el deseo de certidumbre que generan los métodos automáticos y mecánicos, frente a la ansiedad y la incertidumbre que podemos encontrar en métodos múltiples, lentos, vulnerables, modestos, inciertos y diversos como los denomina el autor; ya que el mundo responde a lo que nosotrxs preguntemos y esto puede ser lineal y por lo tanto controlable, o fractal y así mas cercano a las realidades que son complejas, difusas, desordenadas, y a veces inasibles.

Queda claro que "el problema de cientificidad no es solamente un problema teórico, es también un problema metodológico. Esto significa que mi metodología, más que una lista sobre técnicas de recolección de datos, precisa de una discusión teórica, contextualizada y práctica" (Suárez-Krabbe 2011: 188). Esto ha llevado a que las investigaciones feministas y las activistas desarrollen metodologías que rompan con los enfoques hegemónicos. Al respecto, Martha Patricia Castañeda (2019), quien se ha interesado por comprender las perspectivas y aportes de la investigación feminista a la emancipación, afirma que el fortalecimiento de las posturas críticas ha requerido conectar los postulados teóricos con las aproximaciones metodológicas, para ofrecer alternativas frente a los procedimientos estandarizados que reproducen las orientaciones androcéntricas de las metodologías convencionales.

Igualmente, va emergiendo el reconocimiento a la creatividad e innovación de quienes hacen investigación feminista, buscando tejer redes entre quienes están en la academia y quienes generan y sistematizan conocimientos feministas fuera de ese ámbito; así se trae a la academia formas de hacer propias del activismo y, a la vez, se llevan metodologías académicas y científicas más allá de sus límites disciplinares e institucionales. Siguiendo a Castañeda (2019) este emplazamiento teórico-metodológico se caracteriza por ser multi-pluri-interdisciplinar, con abordajes de investigación mixtos, no solo por las combinaciones entre lo cuantitativo y lo cualitativo, sino por la adopción de métodos no convencionales, asociados con las tecnologías de la información y la comunicación y con otros recursos tecnológicos, artísticos y populares que rompen los cánones de los enfoques tradicionales. Para Castañeda esto lo posibilitan investigaciones colectivas,

"lo que implica reflexiones profundas en torno a la titularidad de la investigación, la autoría del conocimiento, el reconocimiento a la autoridad epistémica, la finalidad de la indagación y el uso posterior de los resultados obtenidos. En este campo se ha abierto una franca discusión entre la investigación activista y la investigación académica, discusión en la que la validación de los métodos y los conocimientos obtenidos ocupa un lugar central" (Castañeda 2019: 31).

Estas reflexiones también van a ser abordadas en las experiencias de coinvestigación, que son una de las formas como las investigaciones que se posicio- 
nan como activistas ponen en práctica el replanteamiento sobre las metodologías, métodos y técnicas de investigación (Borio, Pozzi y Roggero 2004). Estas se conciben como actividades de transformación social, lugares de formación y de cooperación diferentes en la búsqueda de producción de conocimientos otros, experimentación de prácticas organizativas y espacios de resubjetivación. La coinvestigación se forja de manera crítica y problematizadora, las certezas posibles deben responder a la experiencia concreta en el terreno y ponerse siempre en discusión. Igualmente, es considerada como acción política que surge desde dentro de los procesos políticos donde se conjuga teoría y práctica, teniendo resonancias en la reinvención de las formas de activismo.

Siguiendo a Borio, Pozzi y Roggero en los ejercicios de coinvestigación van a ser fundamentales los procesos de cooperación de los cuales surgen los conocimientos, siendo éstos productos del trabajo interno y no solo de investigadorxs externxs. Para ello, se van a poner a prueba "metodologías experimentales, flexibles, que puedan constituir modelos abiertos, ponerse en circulación y confrontarse con experiencias distintas" (2004: 67-71). Es por tanto un proceso abierto, donde la relación conocimiento y acción política se mueve en espiral y aunque sean dimensiones distintas no se separan, de allí que se consideren los espacios de coinvestigación como espacios de formación política, proporcionando sistematicidad y eficacia a la acción política.

Existe en este sentido una experiencia concreta de investigación activista feminista denominada producciones narrativas que, basada en la epistemología de los conocimientos situados, pone en práctica una técnica que intenta responder a objetivos académico-políticos en la medida en que permite la construcción colectiva de conocimientos basada en el reconocimiento, valoración y legitimidad de los diversos saberes, posibilitando deconstruir la relación extractivista sujetoobjeto de conocimiento, construyendo saberes que tengan sentido para todas las personas que participan en los procesos.

Las narrativas son parte significativa de nuestras vidas y están ancladas de tal manera que organizan nuestra experiencia, por ello no son una producción individual sino que se encuentran relacionadas con nuestro contexto sociocultural, teniendo efectos en nuestras realidades que pueden ser interpretadas y leídas de distintas maneras. Recoger estas experiencias y sentidos es una de las tareas de las producciones narrativas, con la cual se le da un especial énfasis en la perspectiva de quien participa en su construcción, lo que permite identificar formas de agencia de lxs actores sociales y recoger distintas comprensiones sobre un fenómeno determinado (Pujol y Montenegro 2013). Asimismo, es importante resaltar que la producción narrativa es una

"toma de agencia especialmente en el momento en el que éstas se autoconstruyen como alternativas a las (meta)narrativas dominantes. Su carácter productivo y potencialmente político viene resaltado con el reconocimiento de su parcialidad (debida al posicionamiento situado de quienes las producen) y su temporalidad (abierta a ser modificada con el pasar del tiempo). Se quiere subrayar el carácter construido, constituyente, parcial, político y procesual de las narrativas" (Biglia y Bonet-Martí 2009: 14). 
La producción de narrativas es un proceso que, como afirman Pujol y Montenegro (2013), se debe adaptar a las necesidades particulares de las investigaciones, en coherencia con los principios teóricos y epistemológicos que la guían. Lo relevante también es que se involucra a las personas en la "producción situada de visiones de un fenómeno determinado"; el ejercicio en concreto consiste en:

"la producción conjunta de un texto híbrido construido conjuntamente a partir de a) sesiones donde la investigadora y las participantes hablan y discuten distintos aspectos del fenómeno que se quiere estudiar, b) la textualización, que funcionaría como una revisión y reflexión sobre la sesión, en el que la conversación se traduce a un texto organizado y comunicable que refleja las posiciones y argumentos desarrolladas a lo largo de la sesión, y c) el reconocimiento de la agencia de las participantes para modificar, corregir, expandir la textualización realizada hasta que validen la narrativa creada" (Balasch y Montenegro, citadas en Gandarias 2004: 31).

Es así como las Producciones Narrativas permiten una reflexión constante sobre el proceso, prestando especial atención al pensamiento situado y las responsabilidades políticas que implica, así como la validez epistémica de los diversos conocimientos y el reconocimiento de la agencia de las participantes. Igualmente, posibilita poner en práctica la investigación feminista como "una praxis que liga experiencia y acción. De ahí que se torne central la experiencia, en términos de quién es el sujeto de dicha experiencia qué está siendo representado y validado dentro de la investigación" (Gandarias 2004: 128-129), ya que lo que se busca es construir conocimientos que sean útiles no solo para la investigadora sino para las personas y colectivos con las cuales se construyen, contribuyendo así al impacto de la acción política feminista, esta es justamente la potencia de la propuesta en la medida en que

"otra posibilidad que ofrece las Producciones Narrativas es que permite abrir espacios de producción de conocimiento más allá de la academia. Al tratarse de textos con entidad propia, las narrativas pueden ser utilizadas por las participantes de la investigación para otros fines que no sea únicamente la investigación académica; facilitando puentes de conexión entre la academia y los movimientos sociales" (Gandarias 2004: 132).

Las Producciones Narrativas van más allá de la utilización de una técnica específica para la recopilación de información en la medida en que se convierte en un método interpretado como proceso, es por ello que podemos comprender "la narrativa de forma simultánea como objeto de estudio, método de indagación y producto de investigación. De igual manera, [...] la narrativa puede abordarse a la vez como proceso y como fenómeno. Como proceso o método resulta en una lógica de indagación; como fenómeno representa la estructura cualitativa de la experiencia" (Clandinin; Connelly 1994 citado en Martínez y Montenegro 2014: 115).

Sumado a ello, las narrativas también permiten acceder a formas de comprensión y creación de significados difractarias, en el sentido que propone Donna Haraway (1999), al ser formas de producción de conocimiento que no 
representan la realidad sino que producen nueva teoría y aportan una diversidad de miradas sobre lo investigado, conocimientos que están relacionados con las meta-narrativas, comprendidas como nociones abarcadoras ya definidas. Estas experiencias que difractan permiten reflexionar sobre cómo estas meta-narrativas han sido incorporadas, rechazadas, subvertidas, cuestionadas, complejizadas o desplazadas.

En una investigación activista feminista la pregunta por el cómo resulta crucial en la medida en que una investigación no es feminista simplemente porque la investigadora lo es, o porque trabaja con quienes se consideran a si mismas mujeres, o porque asume las propuestas de las epistemologías feministas. Como no existe un solo método, ni una sola epistemología, o forma de concebir y por lo tanto construir conocimiento feminista -ni es esa la pretensión- la pregunta es: ¿qué hace feminista una metodología, una investigación? y sus implicaciones en la práctica. En este sentido, la apuesta es por poder construir conocimientos colectivos que partan de la experiencia. La propuesta de las producciones narrativas es pertinente para quienes tenemos la intención de poner en práctica la construcción teórica de abajo hacia arriba; además que no es una fórmula ni una jaula metodológica, sino que permite ser adaptada a las características de cada investigación y de las personas que en ella participamos.

En un ejercicio concreto de investigación activista feminista que apuesta por otras formas de construir conocimientos con las producciones narrativas, los retos, dificultades y aprendizajes son diversos, teniendo en cuenta las particularidades de los contextos, las sujetas de investigación y los objetivos propuestos. Acudiendo de nuevo a mi experiencia en este sentido el tiempo fue una de las limitaciones más fuertes, no solo porque los tiempos de la academia y el activismo son diferentes, sino porque es importante reconocer los momentos en los que se encuentran los colectivos y las personas que posibilitan o no el trabajo con este tipo de propuestas de investigación participativas, con metodologías que implican compromisos extras y tiempo para ello.

Considero que este es uno de los límites y a la vez es parte de los retos que implican los procesos de construcción colectiva de conocimiento situado cuando deseamos realizar una investigación activista feminista, porque participar en una investigación con producciones narrativas implica trabajo en todos sus momentos. Una alternativa para activar el compromiso es que los temas o problemas que nos convoquen y las sesiones de trabajo sean pensadas en conjunto para que respondan a los intereses de todas y no solo de lx investigadorx, o que coincidan para que nos entusiasmen a todxs.

\section{IMPLICACIONES POLÍTICAS}

El aporte a la construcción de conocimiento que realizan las epistemologías feministas tienen una estrecha relación con la política, en la medida en que la relación con los movimientos feministas y la militancia activa ha posibilitado que "ante la emergencia de un vacío de conocimiento los problemas planteados por 
las feministas se convirtieran en problemas de investigación” (De Barbieri 1998: 121). Esto se evidencia no solo en la selección de los problemas a investigar, sino en las preguntas que se formulan y las decisiones en cuanto a los resultados, la particularidad de este tipo de investigaciones es que se hace explícito el punto de vista y se sitúa la investigación, de allí su importancia para la investigación activista.

Sin embargo, este tipo de investigaciones se enfrentan con la necesaria superación de la dicotomía entre academia y activismo impuesta como antagónica, que trae consigo tensiones y contradicciones

"En la vida cotidiana esas tensiones siguen evidenciándose discursivamente, por ejemplo, hay quienes desde la academia (ojo no estoy diciendo que "todos los académicos") califican a las investigaciones realizadas por las ONG y los activistas como: "parciales, superficiales, subjetivas, imprecisas, tendenciosas, falsas". Por su parte, hay activistas que lanzan fuertes críticas a las investigaciones académicas y las califican de ser "extractivas", de pregonar una "ficticia objetividad", de ser producidas para el petit comité (o sea, de ser "elitistas"), de ser "poco oportunas" e incluso "inútiles para la gente a la que estudian"” (Leyva 2010: 5).

Esta narrativa sobre como ven y valoran el trabajo desde sus propios lugares de producción de conocimiento, tanto lxs investigadorxs como lxs activistas, se supera con diversas experiencias que han demostrado que hay superposiciones de agendas académico-políticas. En este sentido, han sido fundamentales las vivencias de los feminismos que han articulado a lo largo de su historia los procesos de investigación y activismo feminista, haciendo esfuerzos por no perder la conexión que ello implica entre teoría, análisis de la complejidad y experiencias, gracias a las cuales existen nuevos procesos de producción de conocimiento. De esta manera se concreta la idea que en determinados casos solo se puede llegar a conocer en profundidad si se participa activamente en dicho proceso (Leyva 2010).

Para que esto sea posible, es necesario repensar el lugar de lx investigadorx cuya posición de poder habitual se cuestiona y transforma, en este sentido:

"No estamos delante de la muerte del sujeto (en este caso investigador[a]), como se critica desde una versión moderna, sino más bien nos encontramos con su apertura así como con las aperturas de agentes y territorios narrativos no isomorfos; cosa que permite multiplicar las miradas y obtener una visión más polimórfica de las realidades; entender así algo más de la complejidad en la que nos desenvolvemos" (Biglia 2012: 208).

Siguiendo a Biglia (2012), la transformación del lugar de lx investigadorx pasa por abrir también los vínculos y los lugares donde es posible construir conocimientos, en los cuales confluyen para tejer relaciones diferentes miradas del mundo, perspectivas analíticas, propuestas políticas y acciones que lleven a la práctica los saberes producidos. En este sentido, será importante el impacto que tiene cada subjetividad que se encuentra marcada por las posiciones de vida, 
además de las teóricas y políticas, de quienes participan en las investigaciones activistas feministas, como ya hemos visto que plantea el conocimiento situado.

Otro aspecto relevante es que estas relaciones se construyen, no se preestablecen simplemente, es aquí donde surgen preguntas como hasta dónde llegar cuando lxs investigadorxs se involucran en los procesos políticos organizativos, en el sentido de si es o no necesario poner límites a las maneras como se participa y a los tipos de relaciones que se construyen, igualmente cómo construirlas y qué metodologías de trabajo las posibilitan. Se trata de poner en práctica la opción política de colocarse en el mismo plano crítico con quienes se realiza la investigación, desde un mutuo reconocimiento que posibilita una relación intersubjetiva:

"En esta situación se buscarían la identificación, la comprensión y la implicación como elementos de un proceso que abriría la posibilidad de compartir el mundo desde las posiciones diferenciadas de quien investiga y quien participa en la investigación como poseedora de saberes que pueden dar pie a la producción de un conocimiento. La noción contemporánea de intersubjetividad supone una relación de mutua interpelación en la que, al no objetivar a las personas, se busca que haya respeto, apertura, diálogo y delimitación en las posiciones relativas que cada quien ocupa en la investigación" (Castañeda 2008: 86).

En términos de una investigación activista feminista, la construcción de este tipo de relaciones pasa necesariamente por reconocer la diferencia entre las mujeres con el fin de hacer evidentes las contradicciones que también pueden existir, producto de los posicionamientos diferenciados y las implicaciones de los diferentes sistemas de opresión. Se busca así superar tanto la imagen idealizada de la igualdad entre las mujeres, como la subalternización o victimización en la que se puede situar a las mujeres con quienes se trabaja, ya que "como investigadoras feministas no podemos ilusionarnos/fingir que nuestra posición sea menos influenciada por la historia que otras y debemos delinear líneas de evaluabilidad de trabajos realizados gracias al uso de metodologías feministas" (Biglia 2012: 208).

Al respecto, los feminismos decoloniales (Medina 2019) en su diversidad, nos proponen que el falso dilema entre activismo y academia no es banal, porque nos ayuda a comprender cuáles son lxs sujetxs y los procesos que cuentan o no con una legitimidad epistémica, invitándonos a reflexionar sobre ello. Justamente porque lo epistémico es político, es necesario estar atentxs a las realidades que han sido desahuciadas por la investigación científica. Ello implica otra forma de relación metodológica que recoja de manera íntegra las voces subalternizadas y sus correlaciones entre experiencias, ideas y propuestas, como mecanismo para dialogar y pensar con.

Surge entonces el problema de la representación y la responsabilidad política que ello conlleva, ya que, a pesar de las experiencias positivas en los procesos de investigación con las cuales se apuesta por construir relaciones horizontales, las diversas posiciones de lxs sujetxs involucrados en las investigaciones no son fáciles de deconstruir; en particular por las formas que toman los resultados de 
las investigaciones -libros, tesis, informes, artículos, etc.-, porque son textos que de alguna manera terminan representando a los colectivos y personas con las cuales trabajamos, al ser un medio que los hace visibles y con el que nos acercarnos al conocimiento de sus experiencias, lo que lleva a cuestionar la autoridad de lxs investigadorxs para ello. Además, estas responsabilidades se acrecientan, como afirman Biglia y Jimenéz (2012), cuando en los espacios académicos los procesos colectivos de construcción de conocimiento no son reconocidos como viables, y aún falta por construir mecanismos de validación que transciendan estos espacios, lo que no se puede desligar de las finalidades y usos del conocimiento que se produce.

Toda esta situación se hace mas compleja cuando quien investiga es a su vez unx activista políticx, o quien milita también es unx investigadorx, posición que supera los límites de una y otra actividad que, como hemos visto, se ha intentado mantener separada, de allí su constante cuestionamiento. Se trata de una postura que conjuga posiciones políticas y epistemológicas, en un encuentro entre acciones políticas y saberes para y acerca de estas acciones, procesos de donde surgen tensiones y contradicciones que la investigación activista feminista va a enfrentar.

Sin embargo, surgen otras preguntas como sí es necesario una inmersión plena, un compromiso total con los activismos, para autoras como Maristella Svampa

"el total involucramiento dificulta la reflexión crítica obturando la producción de un tipo conocimiento que vaya más allá de la visión de los actores. A esto hay que añadir que este exceso de involucramiento ha potenciado una actitud de rechazo y de resentimiento hacia el mundo académico, el cual ante los ojos de la sociedad aparece como portador exclusivo del saber ilegítimo" (2008: 172).

Considero que involucrarse plenamente en los procesos políticos no implica necesariamente la imposibilidad de una reflexión crítica, estar adentro, ser y hacer parte, comprometerse políticamente no significa que se olvida el lugar diferenciado desde el que cada unx se posiciona y toma parte. Asimismo, hace parte de la apuesta política dar legitimidad a los conocimientos situados que se construyen en y con los activismos, legitimidad y validez que no solo va a ser importante para el ámbito académico sino principalmente para las personas y los colectivos, para quienes dichos conocimientos van a ser centrales en las propuestas y alternativas frente a lo que se lucha.

Esto no es óbice para reconocer cómo influye aún la distancia que permanece entre la academia y los movimientos sociales, entre lxs investigadorxs y las organizaciones sociales, así seamos activistas feministas y logremos construir confianzas o seamos cercanas. Cuando pensamos nuestro propio activismo es diferente si ello es una iniciativa que viene de afuera, aunque proponga ser participativa, a cuando surge desde dentro de los propios procesos organizativos y sus necesidades, o de las prácticas colectivas o individuales. 
Decidir realizar una investigación activista feminista implica tener en cuenta que nos enfrentamos a estos retos y preguntas, que se trata de superar dicotomías, binarismos enclaustadores, pero también moverse en los márgenes, en las fronteras, en los encuentros y desencuentros, ello implica repensar el activismo y la investigación como continuum. Es en el trabajo concreto donde quizá encontremos respuestas a cómo movernos en los intersticios y articulaciones posibles entre los activismos, las investigaciones y los feminismos, cómo hacer prácticos los aportes que las epistemologías y metodologías feministas traen a las acciones políticas y como éstas a su vez afinan y enfocan las investigaciones en una relación dialéctica.

Quedan por tanto más preguntas abiertas que posibles respuestas, ya que se trata de construir un accionar investigativo activista que no se cierre al ámbito académico, sino que haga uso creativo del mismo politizándolo, para ello es necesario buscar y hacerse un lugar en los activismos basado en el compromiso sin dejar de lado las posturas críticas, para lo cual va a ser fundamental poner en práctica tanto la reflexividad como la difracción de las realidades.

\section{A MANERA DE CONCLUSIÓN: LA REFLEXIVIDAD Y LA DIFRACCIÓN COMO HERRAMIENTAS}

Teniendo en cuenta que los supuestos básicos que caracterizan la investigación activista feminista son: el compromiso para y con el cambio social, la ruptura de la dicotomía público/privado, la relación de interdependencia entre teoría y práctica, el reconocimiento de la perspectiva situada, la asunción de responsabilidades, la valoración y el respeto de la agencia de todas las subjetividades que están implicadas, explícita o implícitamente en el proceso de investigación, la puesta en juego de las dinámicas de poder que intervienen en el proceso, una continua disposición a que quienes hacen la investigación se permitan ser transformadas por el proceso, la reflexividad y autocrítica, los saberes colectivos como reflejo de lógicas no propietarias, y la redefinición de los procesos de validación del conocimiento (Biglia 2007: 416-418).

Realizar una investigación activista feminista pasa por tener claridades frente a nuestra postura identificando el lugar en el que nos posicionamos en el contexto académico, no solo lo que entendemos por conocimiento y ciencia sino de dónde vienen los criterios a partir de los cuales le damos un valor. Es importante hacer evidentes nuestras reflexiones y decisiones sobre los supuestos, las dudas, las certezas y las incertidumbres desde las que partimos y hacia donde llegamos cuando hacemos investigación.

También es necesaria la mirada crítica para comprender en qué se sustenta la autoridad de quienes son reconocidxs como investigadorxs, así como las restricciones institucionales que enfrentamos, las preguntas que son o no permitidas, las maneras de reaccionar frente a las respuestas, las alianzas que son posibles de realizar o no, las formas de trabajo consideradas legítimas. De esta manera la 
reflexividad remite a nuestro lugar tanto frente al ámbito académico como frente a las relaciones que posibilitan las investigaciones.

Se trata de habitar las investigaciones teniendo presente que estos procesos no solo tienen que ver con todas sus etapas, sino con la sensación de poder experimentar un ejercicio de reflexividad sobre las incomodidades que va generando el camino. Desde mi vivencia, es necesario ser conscientes sobre lo que confunde, hace perder el norte -o mejor el sur-, y lo que incomoda. Así se va poniendo el cuerpo en las incertidumbres y descubriendo que las investigaciones no necesariamente tienen que ser lineales, sino que también pueden ser en espiral o en la forma que se vayan dando. Igualmente, se trata de estar atentxs al impacto de nuestras investigaciones, no solo en términos de los resultados sino de todo lo que se va generando durante el proceso, incluidos los encuentros y desencuentros, el tipo de relaciones que se construyen en el camino de la coproducción de conocimientos; lo que pasa por reflexionar sobre el alcance de nuestros compromisos y lo que se va generando tanto en el nivel subjetivo como colectivo, con el fin de hacerse responsable y dar cuenta de ello.

Practicar la reflexividad por tanto es tener miradas críticas y sobre todo autocríticas, así como no dejar de lado lo que las investigaciones suscitan también en términos emotivos, saber que el error es parte del quehacer, tener claridad frente a nuestras posturas y hacerse responsable de sus efectos, estar atentxs también a lo que van generando en diferentes niveles los procesos de co-construcción y participación. Implica ubicar las preguntas no solo en el afuera sino en nosotrxs mismos para no evadir nuestros prejuicios, suposiciones, preconceptos, sino partir de ellos para rehacerlos propositiva y responsablemente; que no solo tiene que ver con pensar las teorías y categorías con las cuales establecemos una conversación y profundizamos en el análisis, sino repensar además nuestra posición política.

Donna Haraway (1999), plantea una opción que permite acercarnos y ver otros elementos que también interactúan en la producción de conocimientos que es circular, abierta, interrelacional. La autora usa la imagen de los rayos que difractan con el fin de producir modelos de interferencia que induzcan cambios en la tecnociencia, para introducir las diferencias que son mas cercanas a nuestras vidas y cuerpos; en coherencia con su propuesta de ser capaces de producir conocimientos que escapen a los dualismos tradicionales -mente/cuerpo, objetivo/ subjetivo, hombre/mujer, sujeto/objeto, etc. -, para ello es necesaria una mirada que más que reflejar difracte, y más que distanciar conecte y articule.

La propuesta de la difracción de Haraway sitúa el conocimiento entre relaciones materiales, políticas y semióticas, cuya funcionalidad no es solo representar la realidad sino articular dichas relaciones. Por lo tanto, si dicha representación como imagen reflejo distanciada no es posible es necesario diferenciarse de lo que consideramos dado para comprenderlo también como construido, esta diferenciación puede ser factible con su propuesta de la difracción con la cual nos posicionamos frente al conocimiento como el mencionado proceso de articulación material, político y semiótico. 
De esta manera, es posible superar la idea de conocimiento como una imagen reflejo de una supuesta realidad lisa para, por el contrario, poder ver las diferencias y cambios de dirección que produce la difracción. El conocimiento entonces puede ser crítico y es siempre político, ya que abre posibilidades que no veíamos en la imagen reflejo, potenciando el cuestionamiento de la supuesta naturalidad y neutralidad de lo dado. De esta manera nos articulamos con la realidad para conocerla sin verla solo como un objeto o un hecho, sino como algo también en constante cambio y construcción, que puede siempre estar en discusión produciendo diversas narrativas.

Sin embargo, permanece la duda sobre cómo permitir que las difracciones nos atraviesen para corporeizarlas en las investigaciones. Una propuesta complementaria en este sentido surge de la resignificación que algunas investigaciones feministas han hecho de la noción de senti-pensar (Salgado 2019), como expresión de una forma de generar conocimiento desde la cosmovisión en la que no hay esa separación sino una concepción de unidad, tanto de cada sujetx, de la colectividad y del cuerpo-pensamiento, en la que ambos procesos se suponen y superponen. Esta noción tiene implicaciones profundas en la investigación, puesto que requieren integrar las múltiples dimensiones que conforman no solo a lxs individuos, sino a sus colectividades y procesos.

En síntesis, todos estos elementos que confluyen en la investigación activista feminista nos ubican en el reto de crear nuevas maneras de producir conocimientos para remover las formas hegemónicas heteropatriarcales y coloniales sobre las cuales aún se sustenta. Ello pasa por estrechar la relación entre producción de conocimientos y prácticas políticas, no separar la investigación de la intervención social, superar las dicotomías metodológicas entre lo cuantitativo y lo cualitativo y las valoraciones que se hacen para su uso en determinados casos.

Asimismo, es importante prestar suficiente atención a los usos reduccionistas, acríticos y perversos de la categoría género, así como a las reales implicaciones de lo que significa hacer investigaciones situadas, cómo ello influye en concreto en nuestras formas de hacer. Se trata de concretar en la experiencia las epistemologías feministas ya que estas no son estáticas, por lo contrario permanentemente nos enfrentamos con su puesta en práctica para construir referentes sobre los cuales las investigaciones activistas feministas y todas las que reconozcan su potencia política se puedan fortalecer.

\section{BIBLIOGRÁFICA}

Biglia, B. (2007): "Desde la investigación-acción hacia la investigación activista feminista", en Perspectivas y retrospectivas de la psicología social en los albores del siglo XXI, Madrid, Biblioteca Nueva, pp. 415-422.

Biglia, B. (2012): "Corporeizando la epistemología feminista: investigación activista feminista", en Subjetivación femenina: investigación, estrategias y dispositivos críticos, Moterrey, Universidad Autónoma de Nuevo León, pp. 195-212. 
Biglia, B.; Bonet-Martí, J. (2009): "La construcción de narrativas como método de investigación psico-social. Prácticas de escritura compartida”, Forum Qualitative Sozialforschung/Forum: Qualitative Social Research, 10(1), Art. 8., disponible en http:// nbnresolving.de/urn:nbn:de:0114-fqs090183. [Consulta: 05-05-2014]

Biglia, B.; Jiménez, E. (2012): Conformidades y disconformidades en habitar los márgenes en la investigación social. Políticas del Conocimiento y Dinámicas Interculturales, Barcelona, CIDOB.

Blazquez, N. (2012): Epistemología feminista: temas centrales. En Investigación feminista: epistemología, metodología y representaciones sociales, México, UNAM, pp. 21-38

Borio, G.; Pozzi, F.; Roggero, G. (2004): "La coinvestigación como acción política”, en Nociones comunes. Experiencias y ensayos entre investigación y militancia, Madrid, Traficantes de sueños, pp. 67-74.

Casas-Cortés, M. I.; Osterweil, M.; Powell, D. (2010): "Fronteras borrosas: reconociendo las prácticas de conocimiento en el estudio de movimientos sociales", en Conocimientos y prácticas políticas: reflexiones desde nuestras prácticas de conocimiento situado, Chiapas, México D.F., Lima y Ciudad de Guatemala: CIESAS, PDTGUSM, UNICACH, pp. 1-22

Castañeda, M. P. (2008): Metodología de la investigación feminista, Guatemala, Fundación Guatemala Centro de Investigaciones Interdisciplinarias en Ciencias y Humanidades y Universidad Nacional Autónoma de México.

Castañeda, M. P. (2019). Perspectivas y aportes de la investigación feminista a la emancipación, en Otras formas de (des)aprender: investigación feminista en tiempos de violencia, resistencias y decolonialidad, País Vasco, Hegoa - SIMREF, pp. 19-40

De Barbieri, T. (1998): "Acerca de las propuestas metodológicas feministas", en Debates en torno a una metodología feminista, México D. F., UAM-X, pp. 103-139.

Feyerabend, P. (1977): Contra o método, Rio de Janeiro, F. Alves.

Gandarias, I. (2014): "Tensiones y distensiones en torno a las relaciones de poder en investigaciones feministas con Producciones Narrativas", Quaderns, 16(1), pp. 127140.

Haraway, D. (1995): Ciencia, cyborgs y mujeres, Madrid, Cátedra.

Haraway, D. (1999): "Las promesas de los monstruos: Una política regeneradora para otros inapropiados/bles", Política y Sociedad, 30, pp. 121-163.

Haraway, D. (2004): Testigo_Modesto@Segundo_Milenio. HombreHembra@_Conoce Oncorratón $®:$ Feminismo y tecnociencia, Barcelona, UOC.

Harding, S. (1998): "Existe un método feminista?", en Debates en torno a una metodología feminista, México D. F., UAM-X, pp. 9-34.

Law, J. (2004): After Method. Mess in Social Science Research, New York, Routledge.

Leyva, X. (2010), “¿Academia versus Activismo? Repensarnos desde y para la prácticateórico-política", en Conocimientos y prácticas políticas: reflexiones desde nuestras prácticas de conocimiento situado, Chiapas, México D.F., Lima y Ciudad de Guatemala: CIESAS, PDTG-USM, UNICACH.

Malo, M. (ed.) (2004): Nociones comunes. Experiencias y ensayos entre investigación y militancia, Madrid, Traficantes de sueños.

Medina, R. (2019): “Aplicaciones metodológicas en feminismos y de(s)colonialidad”, en Otras formas de (des)aprender: investigación feminista en tiempos de violencia, resistencias y decolonialidad. País Vasco, Hegoa - SIMREF, pp. 111-128. 
Pujol, J.; Montenegro, M. (2013): "Producciones narrativas: una propuesta teóricopráctica para la investigación narrativa", en Coloquios de investigación cualitativa desafíos en la investigación como relación social, Córdoba: Socialex, pp. 15-42.

Salgado, M. (2019): Perspectivas y aportes de la investigación feminista a la emancipación, en Otras formas de (des)aprender: investigación feminista en tiempos de violencia, resistencias y decolonialidad. País Vasco, Hegoa - SIMREF, pp. 19-40.

Santos, B. D. S. (2006): A gramática do tempo: para uma nova cultura política. Para um novo senso comum. A ciência o direito e a política na transição paradigmática, Lisboa, Afrontamento.

Suárez-Krabbe, J. (2011): En la realidad. Hacia metodologías de investigación descoloniales, Tabula Rasa, no.14, pp. 183-204

Speed, S. (2011): Forjado en el diálogo: hacia una investigación activista críticamente comprometida, en Conocimientos y prácticas políticas: reflexiones desde nuestras prácticas de conocimiento situado, Chiapas, México D.F., Lima y Ciudad de Guatemala: CIESAS, PDTG-USM, UNICACH, pp. 409-444.

Svampa, M. (2008): "Notas provisorias sobre la sociología, el saber académico y el compromiso intelectual", en Gérard Althabe. Entre dos mundos. Reflexividad y compromiso, Buenos Aires, Prometeo, pp. 163-180. 
